\title{
Evaluación de la institucionalización de la nueva gobernanza en el desarrollo rural en México
}

\section{Assessment of the institutionalization of the new governance in rural development in Mexico}

\author{
Obeimar Balente-Herrera* \\ José María Díaz-Puente** \\ Manuel R. Parra-Vázquez*
}

\begin{abstract}
Institutionality with a new-governance approach is the prevailing paradigm in the international agenda; new governance has economic efficiency as a supreme value. Upon this institutionality rest what has been fostered in rural development in Mexico for more than a decade; this has agreed with the continuity of center-right governments. Thus, in the present research the institutionalization of new governance in rural development in Mexico was analyzed through two case studies: "Alianza para el Campo" compared to a pilot project of multilateral organisms. Results indicate that decentralization remained at the federal states and reached balance of powers between the federal executive and the states; however there was no advancement in the participation of social and private actors, in deregulation and in improving efficacy.
\end{abstract}

Keywords: institutionality, governance, decentralization, participation, efficacy.

\section{Resumen}

La institucionalidad con enfoque de nueva gobernanza es el paradigma dominante en la agenda internacional. La nueva gobernanza tiene como valor supremo la eficiencia económica. Sobre esta institucionalidad descansa lo impulsado en desarrollo rural en México desde hace una década, que ha coincidido con la continuidad de gobiernos de centro-derecha. En la presente investigación se analiza la institucionalización de la nueva gobernanza en el desarrollo rural en México a través de dos estudios de caso: Alianza para el Campo, frente a un proyecto piloto de organismos multilaterales. Los resultados indican que a través de la descentralización se logró un equilibrio de poderes entre el Ejecutivo federal y los estados, pero no se avanzó en la participación de los actores sociales y privados, en la desregulación y la eficacia.

Palabras clave: institucionalidad, gobernanza, descentralización, participación, eficacia.

* El Colegio de la Frontera Sur, San Cristóbal de las Casas, México. Correos-e: obalente@ecosur. mx,mparra@ecosur.mx

** Universidad Politécnica de Madrid, España. Correo-e: jmdiazpuente@upm.es 


\section{Introducción}

El Banco Mundial afirmaba a finales del siglo pasado (2000) que los principales problemas de los países en desarrollo eran un pobre funcionamiento de las instituciones y un modo de gobierno centralizado, vertical y jerárquico, características de lo que Aguilar (2010) denomina vieja gobernanza. En consecuencia y con base en su peso financiero e intelectual, el Banco Mundial impulsó la reforma institucional basada en un enfoque diferente, denominado nueva gobernanza, cuyas características son: un modo de gobierno descentralizado, con mayor participación de la sociedad y una gestión eficaz (Banco Mundial, 2000).

En este contexto, en México, en diciembre de 2001 fue decretada la Ley de Desarrollo Rural Sustentable (LDRS), la cual describe, en el apartado dos la iniciativa: "El proyecto de ley de desarrollo rural establece un sistema de estructuras e instrumentos de planeación para fortalecer el federalismo e incrementar la eficacia de las acciones de desarrollo rural sin generar nuevas cargas burocráticas ...”, más adelante se apunta en el inciso ocho que: “... la Iniciativa se ubica en un terreno realista con el objeto de desarrollar la institucionalidad necesaria para dar soporte a un proceso de diseño estratégico en el que los sectores del campo sean los protagonistas principales" (LDRS, 2001).

El programa con más presupuesto que opera en el marco de la LDRS es Alianza para el Campo (Alianza en adelante), el cual surgió en 1996 y fue alineado con la nueva institucionalidad en 2001. Así, la institucionalidad del desarrollo rural con base en la nueva gobernanza está ensamblada y operando formalmente en México desde 2001; situación que ha coincidido con la continuidad de gobiernos de centro-derecha. Después de una década transcurrida desde la implantación de la nueva institucionalidad debiesen existir avances en la solución de los problemas para la cual fue diseñada; es decir, se debió avanzar hacia una gestión descentralizada, con mayor eficacia y participación de actores sociales y privados.

Las evaluaciones de la escala nacional de los programas de desarrollo rural han sido realizadas por el Fondo de la Naciones Unidas para la Agricultura y la Alimentación, que junto con el Banco Mundial, la Comisión Económica para América Latina, el Instituto Interamericano de Ciencias Agrícolas, el Fondo Internacional de Desarrollo Agrícola, la Agencia Alemana de Cooperación Técnica y la Agencia de los Estados Unidos para el desarrollo Internacional, integran el Grupo Interagencial de Desarrollo Rural-México (GIADR).

A la par de las evaluaciones nacionales, los organismos del GIADR han desarrollado procesos de evaluación local. En efecto, estos organismos realizaron una evaluación de Alianza para el Campo en el municipio de 
Villaflores, Chiapas; los mismos organismos ejecutaron un programa piloto de reconversión productiva en municipios fronterizos del mismo estado, experimento que sirve como referencia del funcionamiento ideal de la nueva institucionalidad del desarrollo rural en México.

Los resultados de las evaluaciones de la FAO en la escala nacional al programa Alianza reportan una baja eficacia -que va de 49 al 58\%-y una débil participación de la sociedad rural en los Consejos de Desarrollo Rural Sustentable (FAO-Sagarpa, 2004 y 2005). Con estos resultados los mismos evaluadores señalan que se "reduce la viabilidad política de la Alianza para el Campo" (FAO-Sagarpa, 2007: 89).

El cuestionamiento de fondo que hace la FAO al programa Alianza implica analizar desde una perspectiva de toma de decisiones a múltiples escalas la institucionalidad para el desarrollo rural en México, pues los actores interactúan bajo reglas determinadas y estas mismas son las que otorgan la capacidad a los programas de desarrollo rural para responder a las necesidades de la gente. Así, el objetivo de la presente investigación es realizar una evaluación de la institucionalización de la nueva gobernanza en el desarrollo rural en México. Para este propósito se utilizó la información de las valoraciones locales desarrollados por GIADR, con lo cual se buscó realizar una investigación profunda, y al mismo tiempo comparar ésta con una experiencia donde se le dio una respuesta creativa al problema de la débil participación y baja eficacia de los programas de desarrollo rural en México.

El documento se integra por un marco teórico donde se analiza la historia y elementos conceptuales de la institucionalidad y la gobernanza, una metodología de estudios de caso comparados y una sección de resultados que inicia con la identificación de los actores estratégicos, para enseguida desarrollar la evaluación mediante criterios que valoran el tránsito de los programas de desarrollo rural desde la vieja hasta la nueva gobernanza.

\section{Marco analítico}

Según Piñeiro (2009) las instituciones son construcciones históricas que responden a demandas, inercias e innovaciones y que crean espacios de decisión dentro de las cuales los actores definen sus cursos de acción y conforman sus patrones de interacción.

El Estado, a través del gobierno, es el actor principal del cambio institucional, ya que es éste quien establece las leyes, así como políticas y programas para fortalecer la capacidad institucional (Prats, 2003; Herrera et al., 2009). Es en este sentido como se realiza el análisis de la historia 
y fundamentos de la institucionalidad del desarrollo rural en los siguientes párrafos.

Los gobiernos latinoamericanos basaron su intervención desde la década del cuarenta hasta los setenta del siglo xx en la vieja gobernanza (Aguilar, 2010). La vieja gobernanza considera débil e incapaz a la sociedad, y es sobre esto que fundamenta un gobierno centralizado, con una organización reglamentada en su actuación, que tiene líneas de mando descendientes, a las cuales se atiende para la consecución de resultados y difusión de información (Aguilar, 2006 y 2010).

En la época de la vieja gobernanza, los gobiernos latinoamericanos realizaron inversiones estratégicas para superar los problemas que obstaculizaban el desarrollo, especialmente en infraestructura y recursos humanos; del mismo modo, fomentaron la producción y regularon los mercados (Piñeiro, 2009). A esta etapa también se le conoce como de sustitución de importaciones.

Para finales de la década de los setenta la mayoría de los estados latinoamericanos se enfrentaron a una crisis fiscal que los obligó al ajuste estructural, el cual consistió en: a) disciplina fiscal encaminada a eliminar los déficit a través de una severa reducción de la burocracia estatal; b) redireccionamiento del gasto público con criterios de rentabilidad y c) una reforma fiscal orientada a ampliar las contribuciones y facilitar su cobro (Piñeiro, 2009); a esta etapa institucional le subyace un enfoque gerencial (Herrera et al., 2009).

A principios de la década de los noventa del siglo pasado, el gobierno mexicano comenzó a establecer un conjunto de normas y decisiones en el sector rural donde prevalece un Estado reducido a la par de un fortalecimiento de las organizaciones económicas y sociales. Desde la perspectiva de Aguilar (2010), dicho periodo correspondió a la gestación de la institucionalidad con enfoque de nueva gobernanza, donde el Estado siguió privilegiando la eficiencia económica, y se reconoce al gobierno como actor importante, pero no el único.

Lo descrito en párrafos anteriores ilustra brevemente la construcción histórica de la institucionalidad del desarrollo rural que tenemos actualmente, la cual está conformada por reglas y normas, formales e informales que establecen los derechos, obligaciones, expectativas y procedimientos para la interacción entre los actores con enfoque de la nueva gobernanza (Appendini, 2002; Prats, 2003; Piñeiro, 2009).

La institucionalidad, según Piñeiro (2009), se va estructurando con la correspondencia entre lo formal e informal, así como entre lo impulsado recientemente y lo establecido a lo largo de muchos ańos, lo cual ya no existe de manera formal pero se mantiene en las prácticas cotidianas de las organizaciones. 
Como se ha señalado, la institucionalidad del desarrollo rural actual en México tiene como piedra angular a la LDRs, la cual, según Herrera $e t$ al. (2009), retoma los grandes principios de la nueva gobernanza. Por la importancia actual del enfoque de la nueva gobernanza es necesario revisar de manera breve su historia y principios.

Políticos y científicos empezaron a hacer un uso más intenso de la palabra gobernanza a partir de 1988, cuando el Banco Mundial señaló que "el problema de África era una crisis de gobernanza" (Pagden, 1998: 7). Este concepto se asoció con políticas y funciones de organismos de cooperación en países en desarrollo (Hewitt, 1998; Grindle, 2007).

Una perspectiva extrema del concepto de gobernanza es la que se acota a la participación y la construcción de consensos entre organizaciones de la sociedad civil, dejando al gobierno con una participación mínima. Dicha perspectiva tuvo cierto auge en la última década del siglo xx con el florecimiento de las organizaciones de la sociedad y civil y movimientos sociales de importancia regional en América Latina, a las cuales las fundaciones y organismos de cooperación internacional dirigieron sus fondos (Barr et al., 2005; Paramio, 1994). Si bien la construcción de la nueva gobernanza pasa por el fortalecimiento de la sociedad civil, existen límites donde se necesita una participación importante del gobierno para superarlos, conclusión a la que se ha llegado después de varios esfuerzos (Kooiman y Van Vliet, 2000; Paramio, 1994).

El concepto de nueva gobernanza en el que se inspira la LDRs es el establecido por el Banco Mundial (2000) y compartida por varios autores (Kooiman y Van Vliet, 2000; Maintz, 2000), que la definen como un nuevo estilo de gobierno caracterizado por la descentralización y una mayor cooperación entre el estado, la sociedad civil y el mercado para la movilización de recursos y capacidades en programas y proyectos que buscan la solución de la problemática en contextos territoriales específicos. En esta posición de la gobernanza se considera al gobierno como un agente de dirección necesario pero insuficiente, tanto para la identificación de los problemas como para su resolución. En consecuencia, el gobierno central establece interacciones con las organizaciones sociales y la iniciativa privada.

Una posición crítica a la perspectiva del Banco Mundial ha sido esbozada por Prats (2003), quien señala que el gobierno no establece relaciones con toda la sociedad, sólo lo hace con actores estratégicos. Los actores estratégicos, según el mismo autor, son aquellos que cuentan con recursos de poder suficientes para impedir o perturbar el funcionamiento de las reglas del juego, la toma de decisiones y la solución de conflictos.

La interacción de los actores estratégicos está condicionada por las reglas del juego que establecen las instituciones; en este sentido, el análi- 
sis de las instituciones nos permite encontrar el fundamento de los patrones de interacción de los actores (gobernanza) (Prats, 2003). Así, el institucionalismo establece las bases que nos permiten entender la gobernanza en contextos territoriales específicos.

En los estudios institucionales es necesario distinguir las instituciones de las organizaciones, y éstas, de las prácticas organizativas (Appendini y Nuijten, 2002). Las organizaciones son entidades construidas que se corresponden con espacios de decisión de los actores; la estructura organizativa se basa en la definición de roles y debe asegurar un desempeño confiable de la organización; por su parte, las prácticas organizativas son las distintas acciones y estrategias de los individuos para sostener y desarrollar su vida cotidiana (Appendini y Nuijten, 2002).

Según Appendini y Nuijten (2002) si se combinan estudios enfocados en las instituciones y organizaciones con las implicaciones en las acciones y estrategias de los individuos para sostener y desarrollar su modo de vida se pueden tener análisis más robustos de la institucionalidad y sus implicaciones en los territorios.

En sintonía con lo anterior, Moran et al. (1998) señalan que es necesario abordar el estudio de la institucionalidad desde múltiples niveles y en un sentido iterativo dentro y entre las escalas.

\section{Metodología}

La investigación se basa en estudios de caso, vistos como proceso de aprendizaje derivados de la experiencia. Los estudios de caso son pertinentes para la presente investigación porque: a) son útiles para el análisis de un fenómeno contemporáneo en su contexto real, especialmente cuando los límites entre el fenómeno y el contexto no son evidentes, tal como los procesos de desarrollo rural; b) su fortaleza es hacer una investigación profunda sobre una experiencia de interés en la que se haya dado una respuesta creativa al problema en cuestión, conservando la visión total del fenómeno (Yin, 1981); c) se puede generar conocimiento y alternativas para escenarios de sistemas similares, aunque no se puede generalizar lo registrado en el sentido estadístico del término (Arzaluz, 2005; Tellis, 1997); d) los estudios de caso aplicados a los fenómenos descritos nos permiten establecer propuestas de mejora con base a las lecciones aprendidas (Yin, 1981).

En la selección de los casos que se comparan en el presente estudio fueron considerados los siguientes criterios generales: escala territorial equivalente (municipios), participación de organismos multilaterales del GIADR, duración del programa, y que la evaluación se haya realizado. En este sentido fueron seleccionados para ser comparados los estudios de caso 
del Consejo Municipal de Desarrollo Rural Sustentable de Villaflores (en adelante Consejo de Villaflores) y el Proyecto de Reconversión de la Agricultura en la Frontera sur de México (Reagri).

El Consejo de Villaflores se creó formalmente el 18 de septiembre de 2003, para este propósito la Secretaría de Desarrollo Rural de Chiapas (SDR) conjuntamente con la Sagarpa, bajo mandato de la LDRS, emitieron la convocatoria y la hicieron llegar a las diferentes organizaciones productivas e instituciones con presencia en el municipio. Para el 2005, un organismo del GIADR, la Agencia de Cooperación Técnica Alemana (GTZ) presentó al Consejo de Villaflores una propuesta para participar en su fortalecimiento organizacional y de integración de evidencias que le permitiera constituirse en un espacio de toma de decisiones descentralizado encaminado al desarrollo rural sustentable del municipio. La propuesta de la GTZ fue aceptada y los autores de este artículo participaron de 2005 a 2007 en un proceso de investigación-acción como facilitadores y sistematizadores de la información generada en 39 reuniones del Consejo de Villaflores, donde intervinieron un promedio de 20 representantes de organizaciones.

Reagri, por su parte, ha sido un programa piloto de los organismos del GIADR y ha servido como referente para los mismos organismos multilaterales y para las dependencias del gobierno mexicano de como debiese funcionar la nueva institucionalidad del desarrollo rural. En Reagri participaron el Fondo Común para los Productos Básicos, el Fondo de Riesgo Compartido para el Fomento de Agronegocios, el Instituto para el Desarrollo Regional A.C., y los Centros de Desarrollo Regional. Reagri se caracterizó por ser un programa descentralizado y desregulado, que buscó incorporar a productores minifundistas con buenas condiciones agroecológicas al mercado de bienes agrícolas. En Reagri los autores del presente artículo participaron en su evaluación, para lo cual utilizaron grupos focales integrados por técnicos y productores, entrevistas en campo a productores y recorridos por plantaciones e invernaderos.

Los criterios con los cuales se desarrolló la evaluación fueron construidos con base en los principios que articulan a la LDRs y los conceptos que señalan diferentes autores como fundamentos de la nueva gobernanza (Banco Mundial, 2000; Kooiman y Van Vliet, 2000; Maintz, 2000; Kaufmann et al., 2010). Dichos criterios fueron: descentralización, eficacia, participación, regulación y estabilidad política.

La descentralización se concibe como la transferencia de la autoridad fiscal, política y administrativa a niveles locales de gobierno (Blair, 2000; Boisier, 2002; Díaz, 2002). La eficacia, por su parte, se valora en función de las metas establecidas contra los resultados alcanzados en los programas evaluados (Project Managment Institute, 2004), mientras que la partici- 
pación se entiende como un proceso a través del cual los actores involucrados, con diferentes perspectivas, capacidades y activos, trabajan en equipo para alcanzar las metas establecidas en un proyecto (Taylor $e t$ al., 1998). En tanto, la regulación está en función de las dependencias y controles administrativos a los que tienen que atender los responsables de programas y proyectos para informar del cumplimiento de metas (Boisier, 2002; Kaufmann et al., 2010). Por último, estabilidad política se analiza en relación a cómo el gobierno atiende a las necesidades de la gente, en particular a los actores estratégicos (Prats, 2003).

Con los criterios definidos se desarrolló la escala de medición considerando la graduación, la discriminación y la validez de los mismos (Sierra, 1998). Así, los criterios tuvieron como valores mínimos las prácticas que corresponden a la vieja gobernanza, mientras que los máximos son los que se espera se apliquen con la institucionalización de la nueva gobernanza (cuadro 1).

\section{Resultados y discusión}

Esta sección se integra con la identificación de los actores estratégicos que participan con sus capacidades y en el marco de las reglas del juego definidas por la nueva institucionalidad. Enseguida se desarrolla la evaluación de los estudios de caso, para lo cual se inicia con la referencia que corresponde en la LDRS a cada criterio, para después pasar a registrar y discutir los resultados. Se termina esta sección con una síntesis gráfica de los resultados de la evaluación de los dos estudios de caso en cuestión.

\subsection{Los actores estratégicos}

Para valorar con una visión amplia los programas, motivo de este estudio, es necesario identificar los actores estratégicos que interactúan y sus funciones, tal y como lo sugiere Prats (2003). Por este motivo retomamos a Friedmann (2001) y Cazorla et al. (2004), quienes nos señalan que en los ámbitos de la política pública participan cuatro grandes grupos de actores: social (familias y organizaciones de la sociedad civil), privado empresarial (organizaciones formales e informales dedicadas a actividades económicas de iniciativa privada), política (todas las organizaciones políticas formales de diferentes niveles) y público administrativo (administraciones públicas existentes y acciones de iniciativa pública). $\int$

Haciendo uso de las categorías de Friedmann (2001) y Cazorla et al. (2004) es posible identificar que en Alianza participaron: a) en el ámbito político, las cámaras de diputados y senadores, que autorizan los presupuestos cada año; b) en el ámbito público administrativo, la Sagarpa quien 


\section{Cuadro 1}

\section{Criterios de evaluación de institucionalización de la nueva gobernanza}

\begin{tabular}{|c|c|}
\hline Criterios & Escala \\
\hline Descentralización & $\begin{array}{l}\text { 1. Centralizado } \\
\text { 2. Se crean organismos con funciones consultivas, pero no decisorias. } \\
\text { 3. Se crea una organización distinta a la cual se le transfiere capaci- } \\
\text { dad de toma de decisiones y recursos financieros (Boisier, 2002; } \\
\text { Díaz, 2002). }\end{array}$ \\
\hline Eficacia & $\begin{array}{l}\text { La eficacia se valora en función de metas comprometidas/resultados } \\
\text { alcanzados. } \\
1 .<=50 \% \\
\text { 2. } 51-75 \% \\
\text { 3. } 76-100 \%\end{array}$ \\
\hline Participación & $\begin{array}{l}\text { Participación es colaboración y está basada en: } \\
\text { 1. Comunicación: donde el intercambio de información está diri- } \\
\text { gido por lo que al gobierno le interesa transmitir. } \\
\text { 2. Contribución: se basa en la provisión de recursos desde los di- } \\
\text { ferentes actores para soportar el proyecto. } \\
\text { 3. Cooperación: donde los actores involucrados negocian recursos } \\
\text { y asumen responsabilidades para el buen desarrollo del proyecto. }\end{array}$ \\
\hline Regulación & $\begin{array}{l}\text { 1. Regulación centralizada y precisa } \\
\text { 2. Regulación descentralizada a organismos locales } \\
\text { 3. Desregulada }\end{array}$ \\
\hline $\begin{array}{l}\text { Estabilidad } \\
\text { política }\end{array}$ & $\begin{array}{l}\text { 1. El gobierno atiende a las grandes organizaciones de manera } \\
\text { clientelar y autoritaria. } \\
\text { 2. El gobierno atiende a las grandes organizaciones en mesas de } \\
\text { negociación. } \\
\text { 3. El gobierno establece espacios de diálogo entre grandes organi- } \\
\text { zaciones, pequeńos grupos de trabajo y microempresarios. }\end{array}$ \\
\hline
\end{tabular}

Fuente: Elaboración propia.

publica anualmente las reglas de operación del programa, negocia los convenios de colaboración y mezcla de recursos con los gobiernos de los estados de la federación, y la Secretaría de Desarrollo Rural de cada estado de la federación que lleva a cabo la operación de los fondos; los Consejos de Desarrollo Rural Sustentable, en este caso en el nivel municipal, son los organismos que, según la ley, deben priorizar y dictaminar los proyectos de desarrollo rural a llevarse a cabo en el municipio; c) los actores sociales y privados que participan son los grupos de productores y empresarios agrícolas que hacen la gestión de sus proyectos en las ventanillas dispuestas por el Estado.

Reagri, por su parte, constituye una iniciativa que tiene el propósito de coordinar acciones para impulsar la reconversión productiva con la participación de los siguientes actores estratégicos: a) en el ámbito político, el Fondo Común para los Productos Básicos de las Naciones Unidas 
(CFC), el cual establece las bases para la cooperación al desarrollo; b) en el ámbito público administrativo, el Fideicomiso de Riesgo Compartido para el Fomento de Agro negocios de la Secretaría de Agricultura de México (Firco), quien desarrolló los criterios y lineamientos para la operación del programa Reagri; c) en los actores social y privado que participan está el Instituto para el Desarrollo Regional A.C. (Idear) quien fue la agencia de desarrollo que operó Reagri, la cual trabajó en las microregiones años antes a la implantación del proyecto en acciones de: constitución de los grupos, identificación de las comunidades monitor, evaluación de condiciones agroecológicas y en el consenso del proyecto social; los Centros de Desarrollo Regional (que agrupan a las organizaciones participantes en el proyecto) que desarrollaron actividades de organización, reglamentación de las acciones, preparación de los terrenos, establecimiento de los proyectos, monitoreo y evaluación.

\subsection{Evaluación con criterios de gobernanza}

\subsubsection{Descentralización}

La LDRS establece en su artículo 23:

El federalismo y la descentralización de la gestión pública serán criterios rectores para la puesta en práctica de los programas de apoyo para el desarrollo rural sustentable. Los convenios que se celebren entre el Gobierno Federal, los gobiernos de las entidades federativas y de los municipios, se ajustarán a dichos criterios y conforme a los mismos determinarán su corresponsabilidad en lo referente a la ejecución de las acciones vinculadas al desarrollo rural sustentable.

Los Consejos municipales de desarrollo rural sustentable (Comudrs) pueden operar de manera descentralizada en la medida que cumplan con: 1) constitución formal del Comudrs; 2) un plan de desarrollo rural validado por el Comudrs; 3) un departamento de desarrollo rural o equivalente, cuyo responsable haya sido validado por el Comudrs; 4) un programa anual de desarrollo rural del ejercicio fiscal correspondiente validado por el Comudrs, y 5) firmar el anexo de ejecución municipalizada del programa de desarrollo rural junto con la delegación de Sagarpa y el gobierno estatal (Sagarpa, 2005).

El Consejo de Villaflores se constituyó formalmente desde 2003, sesionó periódicamente y de manera sistemática de 2005 a 2007. Como parte de los acuerdos del consejo sus miembros desarrollaron seis talleres participativos microregionales para integrar el plan municipal de desarrollo rural sustentable, el cual estuvo terminado en marzo de 2006. El documento referido recibió observaciones por parte de la Unidad Ope- 
rativa de la Secretaría de Desarrollo Rural del Estado de Chiapas. Las correcciones fueron integradas por los consejeros, con lo cual fue aprobado el plan. Todo este proceso anterior fue encabezado desde el inicio por el responsable del área de desarrollo rural del municipio (Parra y Herrera, 2006).

El trabajo desarrollado por el Consejo de Villaflores le acreditaba haber cumplido los tres primeros pasos para la operación municipalizada, y dado el visto bueno por las instancias correspondientes se podían cubrir rápidamente los dos pasos administrativos restantes. Por tal, en 2007 acudieron comisiones del consejo para hacer la gestión de la descentralización de los recursos. Del mismo modo se aprovechó la visita de un senador de la república y de funcionarios estatales al consejo para solicitar la descentralización. Sin embargo, la respuesta fue negativa en todos los casos, disimulada con postergaciones (Parra y Herrera, 2006).

Sagarpa opera Alianza de manera descentralizada con los gobiernos de los estados de la federación mexicana, así cada año celebra convenios de colaboración (reglas de operación y recursos financieros) entre estos dos niveles de gobierno (FAO-Sagarpa, 2005). En situaciones que el gobierno federal ha tratado de centralizar de nuevo el ejercicio de algunos recursos ha recibido una respuesta contestataria de la bien organizada Conferencia Nacional de Gobernadores (Conago), tal y como se dio en la sesión de Campeche de 2008 (Conago, 2008). Con esto se evidencia que los gobiernos de los estados asumen y defienden la toma de decisiones y operación federalizada de los programas de desarrollo rural.

Por su parte, Reagri a través de Idear recibió los recursos directamente de los organismos centrales, sin pasar por los gobiernos de los estados, y operó los recursos del proyecto de reconversión productiva de acuerdo a normas de CFC-Firco (Parra y Herrera, 2005).

Con los elementos citados y de acuerdo a la escala de medición desarrollada, Alianza está en un nivel intermedio, ya que como está funcionando actualmente, respeta el marco de descentralización al nivel de los estados de la federación de México, lo que ha propiciado un creciente protagonismo de las secretarías de desarrollo rural de los estados (GIADR, 2007). Pero la descentralización no ha llegado al nivel de las regiones y municipios, tal y como lo establece la LDRs. Al respecto, Bodemer et al. (2006) señalan que el viejo federalismo latinoamericano institucionalizó equilibrios de poder entre las elites nacionales y regionales. Por su parte, Reagri se ubica en el nivel tres de este criterio, ya que un ente distinto del organismo central operó los recursos de manera descentralizada y desregulada (Boisier, 2002). 


\subsubsection{Eficacia}

La Ley de Desarrollo Rural Sustentable en su inciso dos de la descripción como iniciativa señala: "El proyecto de ley de desarrollo rural establece un sistema de estructuras e instrumentos de planeación para fortalecer el federalismo e incrementar la eficacia de las acciones de desarrollo rural sin generar nuevas cargas burocráticas...” (LDRs, 2001).

El Consejo de Villaflores no operó proyectos de manera directa debido a que no se logró la descentralización hasta el nivel municipal. De esta manera, no se puede valorar la eficacia para el Consejo de Villaflores.

Alianza, operado desde los gobiernos de los estados, (FAO-Sagarpa, 2005 y 2007), tiene una eficacia que va de 49 a 58\% respecto a metas financieras; la misma FAO señala en los informes de evaluación, que existen demoras considerables en la operación del programa, explicados en parte por la complejidad del mismo. Esta ineficacia compromete incluso la viabilidad política del programa, y por tanto, la gobernanza en las zonas de intervención (FAO-Sagarpa, 2007). La ineficacia se explica porque en cada gobierno de los estados de la federación se ha establecido una burocracia para la operación de los programas equivalente a la que tiene Sagarpa, es decir, se ha generado nueva burocracia y por tal se contradice a lo establecido en la LDRS.

En contraste, la empresa de servicios profesionales que operó Reagri logró una eficacia del 100\% en las metas de implantación del proyecto (hectáreas reconvertidas, viveros, invernaderos, microregiones, organizaciones monitor, organizaciones réplica), mientras que en las metas que corresponden al desarrollo del capital social (fortalecimiento de organizaciones, funcionamiento de redes) tiene avances, pero no alcanzan la consolidación (Parra y Herrera, 2005).

En Reagri la empresa de servicios logró movilizar de manera eficaz los recursos y capacidades de la cooperación internacional y del Estado (enfoque descendente), parcialmente lo consiguió desde los productores y estuvo ausente al inicio del programa la participación de la sociedad civil (enfoque ascendente).

Los autores en visita reciente (17 de julio de 2009) al proyecto Reagri registraron una participación creciente de la iniciativa privada en servicios para la provisión de insumos, asistencia técnica y comercialización; al mismo tiempo que las organizaciones sociales evidenciaban mayores capacidades. Por tanto, se puede decir que no es necesario que todos los actores participen en todas las fases con igual intensidad, pero el trabajo eficaz de unos actores en las primeras etapas facilita la participación de otros en fases posteriores. 
Con la información citada, Alianza se ubica en el nivel uno en la escala desarrollada para este criterio, que corresponde a menos de $50 \%$ de eficacia, ya que el equilibrio de poderes entre la federación y los estados ha dado como resultado una operación ineficaz de los programas de desarrollo rural, situación que no es exclusiva de México, sino de todos los países latinoamericanos (Bodemer et al., 2006). Por su parte, Reagri se ubica en el nivel tres, que corresponde a $75-100 \%$ de eficacia, y se explica en una operación donde confluyeron entes centralizados e instancias locales, sin pasar por organismos intermedios (estados de la federación), lo cual es atípico para los procedimientos formales en México, pero resultó eficaz. Otros programas de desarrollo rural han desarrollado esquemas parecidos de descentralización, tal como lo fue LEADER para Europa, con resultados que suman a la eficacia (Cazorla et al., 2005; AEIDL, 1995). ${ }^{1}$

\subsubsection{Participación}

La LDRs establece las reglas del juego para la participación de los actores en su artículo 24:

Con apego a los principios de federalización, se integrarán Consejos para el Desarrollo Rural Sustentable, homologados al Consejo Mexicano, en los municipios, en los Distritos de Desarrollo Rural y en las entidades federativas. Los convenios que celebre la Secretaría con los gobiernos de las entidades federativas preverán la creación de estos Consejos, los cuales serán además, instancias para la participación de los productores y demás agentes de la sociedad rural en la definición de prioridades regionales, la planeación y distribución de los recursos que la Federación, las entidades federativas y los municipios destinen al apoyo de las inversiones productivas, y para el desarrollo rural sustentable conforme al presente ordenamiento.

El Consejo de Villaflores sesionó regularmente desde 2003. Con una nueva administración municipal en 2005-2007 y la participación de GTZ en acciones de fortalecimiento, se sumaron actores que participaron activamente en 39 reuniones del Consejo de Villaflores, con un promedio de 20 representantes de organizaciones. Del mismo modo se realizaron seis talleres microregionales que dieron oportunidad de participación a los productores en la integración del plan municipal. Todo este proceso de movilización social demostró voluntad desde las organizaciones socia-

\footnotetext{
${ }^{1}$ LEADER: Relación entre acciones de desarrollo de la economía rural. LEADER nace en 1991 en la Unión Europea con base en políticas de diversificación de la economía rural y participación de la gente de las comunidades en la búsqueda de sus propias soluciones. Después de 15 años de funcionamiento de LEADER se considera a éste como "el último gran escalón en la evolución del desarrollo rural en la UE, marcando el inicio de una nueva concepción de la política de desarrollo rural basada en un enfoque territorial, la creación de estructuras de gobierno locales y una gestión descentralizada" (Cazorla et al., 2005: 11).
} 
les para participar y lograr concertaciones, del mismo modo demostraron capacidad para desarrollar criterios e indicadores locales para la aprobación, monitoreo y evaluación de proyectos. Desgraciadamente, la participación disminuyó en 2008 después que no se logró la descentralización de recursos financieros y, por tal, acota al Consejo de Villaflores a funciones de consulta (Parra y Herrera, 2006).

Para el Consejo de Desarrollo Rural estatal de Chiapas, Vázquez (2007) registró que funciona de manera protocolaria y por ello las decisiones sobre las prioridades de inversión en el desarrollo rural se toman de manera autoritaria. Sin embargo, esta situación no sucede solamente en el estado de Chiapas, Según Da Silva (2006) en los Consejos de Desarrollo Rural Sustentable de toda la federación, la participación es cada vez más escasa y su utilidad es cuestionada debido a que se les ha descentralizado funciones pero no la toma de decisiones sobre los recursos de la Alianza.

En contraste, en Reagri, se registró que la participación va incrementando en número de actores conforme las fases del programa avanzan. Al inicio del programa, los productores limitaron su participación a la facilitación de información, conforme los compromisos del gobierno con el programa se hicieron en tiempo y forma, los productores fueron incrementando su colaboración desde: a) aportar ideas, b) tomar iniciativas, hasta c) aportar y arriesgar recursos, así como ser corresponsables con el proyecto (Parra y Herrera, 2005). En este sentido, más participación es mayor compromiso para los productores, lo que al mismo tiempo, según Stalker (2005), lleva a incrementar la posibilidad de éxito de los programas de desarrollo rural.

En la fase de monitoreo, las cooperativas de productores nombraron a las personas para el control y vigilancia de las actividades que corresponden a la implementación del proyecto, lo cual consistía en recorrer cada una de las predios de los socios. Así, la iniciativa de evaluación ya no correspondió a los actores externos, como lo fue en las primeras dos fases del proyecto (Parra y Herrera, 2005).

El programa además de llevar a crear nuevas estructuras, también generó acuerdos y compromisos de los socios de las cooperativas (institucionalidad), las cuales son necesarias para que el programa continúe su buena marcha, pero también para ir consolidando las recientes organizaciones.

Las organizaciones participantes en el programa de las cuatro microregiones se conformaron, en su mayoría, con la facilitación de los técnicos de Idear, como actividad inicial del programa. También participaron en el proyecto organizaciones que ya existían desde hace varios años y con lazos fuertes con organizaciones regionales (Parra y Herrera, 2005).

Con la información registrada, Alianza se ubica en el nivel uno de la escala de medición, que corresponde a una colaboración basada en el 
intercambio de información de acuerdo con los intereses del gobierno; mientras que el proyecto Reagri se ubica en el nivel tres, que significa una cooperación basada en la colaboración donde los actores involucrados negocian recursos y asumen responsabilidades para el buen desarrollo de los proyectos del programa. La forma de participación de los implicados en el proyecto Reagri es una opción que funciona en el contexto local (cultural, social y político), y que nos permite identificar elementos que merecen ser analizados para su implantación en Alianza; por ejemplo, comenzar con una participación que tenga representatividad, pero también operatividad que permita tomar decisiones y ejecutar con eficacia; una operación efectiva permitirá poner en marcha el programa con los tiempos y formas comprometidos; los buenos resultados incrementarán la participación en número y compromiso.

\subsubsection{Regulación}

La norma de operación de Alianza 2011 señala 10 grandes pasos en su esquema de procedimiento de uso de recursos del erario (Diario Oficial de la Federación, 2011: 94). Pero no toma en cuenta los pasos intermedios, ni los que adicionan en cada estado de la federación, que según Cartagena et al. (2005: 11) suman "70 pasos y un promedio de 300 días para que un proyecto de Alianza sea autorizado y entregado los recursos a los productores en el estado de Chiapas".

Los beneficiarios de proyectos de Alianza deben atender controles administrativos de los gobiernos estatales y federales; las normas de operación de Alianza cambian de forma todos los años, por ejemplo la actual fue publicada en diciembre de 2010 y reformada en mayo de 2011, pero no ha mejorado en la simplificación, por lo que el proceso de operación de Alianza sigue siendo centralizado y preciso, acorde a la vieja gobernanza (Cartagena et al., 2005; FAO-Sagarpa, 2007).

Por su parte, la operación de Reagri se puede ubicar como desregulada, ya que atendió una sola dependencia federal y sus controles administrativos, reforzada con la participación creciente de los productores en el monitoreo del proyecto (Parra y Herrera, 2005).

Así, Alianza se ubica en el nivel uno de la escala de medición desarrollada, que implica una regulación centralizada y precisa, en tanto que Reagri se ubica en el nivel tres de la escala, que corresponde a operación desregulada. 


\subsubsection{Estabilidad politica}

Para este criterio no existe cita explícita en la LDRS, sin embargo el secretario de Desarrollo Rural en la exposición de justificación de las reglas de operación 2011 de Alianza, hecha en el Consejo Mexicano para el Desarrollo Rural, enfatiza un proceder incluyente de los diversos actores que puedan aportar soluciones a la estrategia de desarrollo territorial (Consejo Mexicano para el Desarrollo Rural Sustentable, 2010).

El Consejo de Villaflores atendió la estabilidad política con la inclusión de representantes de organizaciones sociales y productivas, a la par de representantes de los ejidos y comunidades. Si bien existen algunas diferencias entre los representantes citados, prevalecieron los acuerdos para evitar duplicar padrones en solicitudes validadas por el Consejo para ser enviadas a diferentes dependencias (Parra y Herrera, 2006).

En el actual y pasado gobiernos del estado de Chiapas se mantiene la estabilidad política a través de la atención directa y sin pasar por el Consejo de desarrollo rural estatal a las grandes organizaciones sociales, las cuales en caso de no atender sus demandas pueden movilizarse y afectar la estabilidad política. Por su parte, el gobierno federal atiende el criterio de estabilidad política a través de la inclusión de las organizaciones del Congreso Agrario Permanente en el Consejo Mexicano de Desarrollo Rural Sustentable, tal y como se evidencia en la lista de participantes y en las actas de sesión del Consejo Mexicano (Consejo Mexicano de Desarrollo Rural Sustentable, 2010)

En cambio, en Reagri se atendió al criterio de estabilidad política considerando a organizaciones sociales y productivas con capacidad de movilización en las regiones de trabajo, al mismo tiempo que los técnicos trabajaron en la integración de otras organizaciones productivas. Esta forma de atender el criterio de estabilidad política en Reagri permitió la inclusión de pequeños productores dispersos y al mismo tiempo crear un contrapeso a las organizaciones sociales tradicionales. Las opciones de ingresos y empleo que generó Reagri han abonado a la estabilidad políti$\mathrm{ca}$, sin que eso signifique ausencia de diferencias entre los actores (Parra y Herrera, 2005). El avance del proyecto de Reagri trae nuevos puntos de conflicto que necesitan solucionar entre los varios actores, tal como la ubicación de las instalaciones de selección, empaque y carga, que todas las organizaciones disputan porque les queden cercanas.

En resumen y de acuerdo a la escala desarrollada para el criterio de estabilidad política, Alianza, operado desde el Consejo de Villaflores alcanza un nivel tres en la escala, del mismo modo lo logra Reagri. Sin embargo, como al Consejo de Villafores no se le descentralizó recursos y quien realmente opera Alianza es el gobierno del estado, esta instancia 
maneja la estabilidad política de acuerdo a lo establecido en el nivel uno (El gobierno atiende a las grandes organizaciones sociales de manera clientelar y autoritaria). En efecto y de acuerdo a lo citado, los gobiernos federal y estatal operan Alianza en atención al criterio de estabilidad política considerando el grado de organización del actor, el acceso a niveles decisorios y la posibilidad de que se genere un conflicto social y político en caso de no atender sus demandas, tal y como señala Ruiz (1996) que son los criterios de diseño de políticas públicas en el formato de la vieja gobernanza. Los actores estratégicos no han avanzado para establecer una mesa de negociación con mayor inclusión, tal y como se espera en la nueva gobernanza. Es importante señalar que la forma de manejar la estabilidad política le ha sido efectiva hasta ahora al gobierno del estado y federal, ya que no se han presentado hasta ahora movilizaciones sociales que le exijan un cambio.

\section{Figura I}

\section{Resultados de la evaluación de Alianza y Reagri}

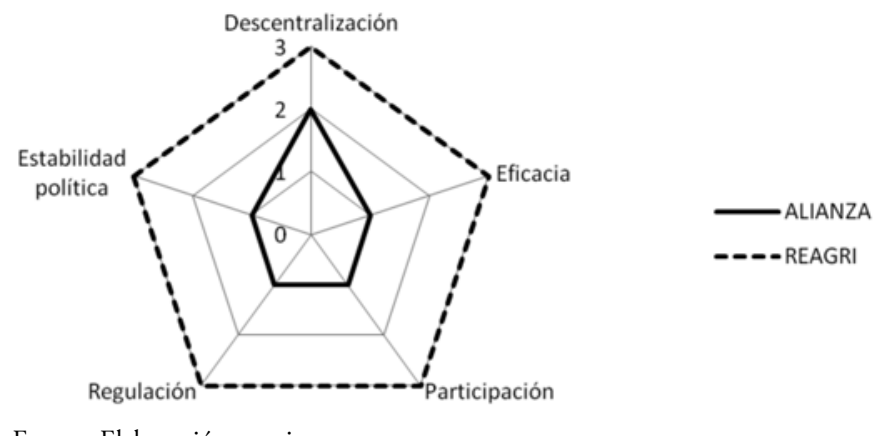

Fuente: Elaboración propia.

\section{Conclusiones}

Los actores estratégicos del desarrollo rural en México aplican el programa Alianza para el Campo con prácticas de la vieja gobernanza, disimulado con la institucionalización de la nueva gobernanza en la Ley de Desarrollo Rural Sustentable.

La descentralización de los programas de desarrollo rural se ha quedado en el nivel de los estados de la federación, con lo cual se ha logrado el equilibrio de poderes entre dos actores estratégicos importantes, de diferentes niveles, pero gubernamentales ambos.

Lo que no se ha logrado con la institucionalización formal de la nueva gobernanza es aumentar la participación de las organizaciones sociales y privadas, el Estado sigue siendo el actor más importante, aunque insu- 
ficiente para identificar los problemas y resolverlos. Esto se ha traducido en ineficacia y nulo impacto en la disminución de la pobreza.

A los gobiernos estatal y federal les funciona, hasta ahora, la atención clientelar y corporativista de las grandes organizaciones para mantener la estabilidad política, y mientras les resulte esta manera de atender las necesidades de estos actores estratégicos no se darán grandes cambios en la descentralización y participación de otros actores.

Los organismos multilaterales a través de los condicionamientos vinculados a los financiamientos para los países, en este caso México, pueden propiciar cambios en las normas y en las relaciones de los actores a diferentes niveles.

Un nuevo actor con capacidades para considerarse estratégico y que demande participar bajo nuevas interacciones y espacios puede también impulsar un cambio en la situación de la institucionalidad actual. Uno de ellos puede ser las organizaciones que integran cientos de municipios en el país. La gestión de descentralización de un municipio de manera aislada no tendrá resultados, tal como fue para el municipio de Villaflores, Chiapas.

Los consejos municipales son un espacio privilegiado para la participación de los actores social y privado, tal como se registró en el Consejo de Villaflores, pero necesita que se le descentralicen recursos para que sea un espacio de toma de decisiones y no sólo de consulta.

Mientras que en el consejo federal y desde los gobiernos de los estados sólo participan como actores estratégicos las grandes organizaciones sociales, en los consejos municipales y en el nivel que se aplicó Reagri tienen cabida pequeñas organizaciones, representantes comunitarios y microempresarios. Las escalas sí importan en la descentralización, ya que se puede valorar con los mismos criterios de la gobernanza tradicional a los actores estratégicos pero la medida es diferente, por tanto, participan un mayor número de actores.

Por lo citado, los retos a superar en la institucionalidad del desarrollo rural, manteniendo la perspectiva de nueva gobernanza, es avanzar en la reglamentación de la LDRS respecto a la descentralización y en este sentido definir las funciones y contribuciones de cada actor en cada uno de los niveles en la estructura organizativa, que propicie una operación con mayor eficacia y facilite una mayor participación de actores sociales y privados.

La forma en que fue operado Reagri constituye una opción en la medida que la descentralización se acompañe de consejos participativos (estatales y municipales) en la toma de decisiones, monitoreo y evaluación de proyectos estratégicos, los cuales pueden ser operados por empresas de profesionales del desarrollo rural. 
En la medida que los gobiernos de los estados y la federación permitan la participación de la sociedad en la toma de decisiones y monitoreo de proyectos, tal y como la nueva gobernanza lo concibe, puede disminuir la excesiva regulación actual que enfatiza en lo documental y soslaya los resultados en campo.

Los retos a superar en la institucionalidad del desarrollo rural en México se enmarcan en un contexto de toma de decisiones a múltiples escalas con creciente importancia de los acuerdos y regímenes supranacionales, que se suman a las condicionantes establecidas por los financiadores internacionales, pero también con la exigencia de participación de un mayor número de actores en formatos más complejos de negociación, dada la intervención de representantes de diferentes jurisdicciones (municipios, estados, federación) en el diseño e implementación de políticas públicas.

\section{Anexo 1 \\ Siglas y acrónimos utilizados}

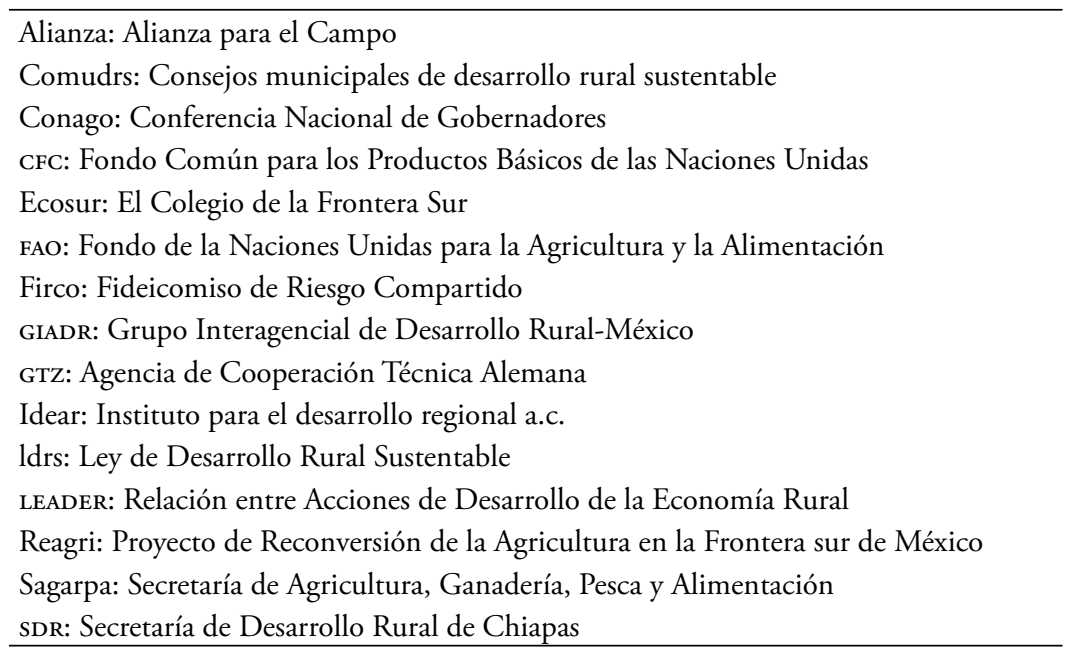

Fuente: Elaboración propia.

\section{Bibliografía}

AEIDL (Observatorio Europeo de Innovación y Desarrollo Local) (1995), "La constitución del proyecto de desarrollo local: la experiencia LEADER", Cuadernos LEADER, Dirección General de Agricultura de la Comunidad Europea, Bruselas, pp. 1-15.

Aguilar, Luis (2006), Gobernanza y gestión pública, Fondo de Cultura Económica, México. 
Aguilar, Luis (2010), "El futuro de la gestión pública y la gobernanza después de la crisis”, Frontera Norte, 22 (43), El Colegio de la Frontera Norte, Tijuana, pp. 187-213.

Appendini Kirsten y Monique Nuijten (2002), "El papel de las instituciones en contextos locales", Revista de la CEPAL 76, Comisión Económica para América Latina y el Caribe, Santiago de Chile, pp. 71-88.

Arzaluz, Socorro (2005), "La utilización del estudio de caso en análisis local", Región y sociedad, xvII (32), El Colegio de Sonora, Hermosillo, pp. 107-144.

вм (Banco Mundial) (2000), Reforming public institutions and strengthening governance: a world bank strategy, World Bank, Washington DC.

BM (Banco Mundial) (2004), La pobreza en México: una evaluación de las condiciones, tendencias y estrategia del gobierno, Banco Mundial, Washington.

Barr Abigail, Marcel Fafchamps and Trudy Owens (2005), "The governance of non-gubernamental organization in Uganda", World Development 33 (4), Elsevier, Oxford, pp. 657-679.

Bodemer Klaus, Joan Prats y Laurence Whitehead (2006), “Manifiesto de la red Latinoamericana de gobernabilidad para el desarrollo ante la cumbre América Latina-Unión EuroEuropea”, en Binneti Carlo y Fernando Carrillo, ¿Democracia con desigualdad? Una Mirada de Europa hacia América Latina, Banco Interamericano de Desarrollo, Washington, pp. 23-29.

Boisier, Sergio (2002), "La odisea del desarrollo territorial en América latina. La búsqueda del desarrollo territorial y de la descentralización", documento preparado para los Seminarios Descentralización de sectores sociales: Nudos críticos y alternativas, Ministerios de la Presidencia, de Educación, y de Salud del Perú, 9-11 de abril, Lima.

Blair, Harry (2000), "Participation and accountability at the periphery: democratic local gobernance in six countries", World Development 28 (1), Elsevier, Oxford, pp. 21-39. 
Cartagena, Pamela, Manuel Parra, Araceli Burguete y Antonio López (2005), "Participación social y toma de decisiones en los Consejos Municipales de Desarrollo Rural Sustentable de Los Altos de Chiapas", Gestión y Política Pública XIv (2), Centro de Investigación y Docencia Económica, México, pp. 341-398.

Cazorla, Adolfo, Ignacio de los Ríos y José Díaz Puente (2005), “La iniciativa comunitaria LEADER como modelo de desarrollo rural: aplicación a la región capital de España”, Agrociencia 39, Colegio de Postgraduados en Ciencias Agrícolas, Texcoco, pp. 697-708.

Cazorla Adolfo, Ignacio de los Ríos y Miguel Salvo (2004), Trabajando con la gente. Modelos de planificación para un desarrollo rural y local, Universidad Politécnica de Madrid, Madrid.

Conago (Conferencia Nacional de Gobernadores) (2008), "Declaratorias 2008”, Secretaría Técnica al Servicio de los Gobiernos de los Estados A.c., México.

Comudrs (Consejos municipales de desarrollo rural sustentable) (2010), "Acta de la primera sesión extraordinaria 2010", Consejo Mexicano de Desarrollo Rural Sustentable, México.

Da Silva, Arilson (2006), Proyecto de evaluación de Alianza para el Campo 2005, análisis prospectivo de política para el desarrollo rural, FAOSagarpa, México.

Díaz, Manuel (2002), "Federalismo fiscal y asignación de competencias: una perspectiva teórica", Economía, Sociedad y Territorio, vol. III, núm. 11, El Colegio Mexiquense, Zinacantepec, pp. 387-407.

FAO-Sagarpa (Fondo de las Naciones Unidas para la Agricultura y la Alimentación-Secretaría de Agricultura, Ganadería, Pesca y Alimentación) (2004), Evaluación Alianza para el Campo 2004, México.

FAO-Sagarpa (Fondo de las Naciones Unidas para la Agricultura y la Alimentación-Secretaría de Agricultura, Ganadería, Pesca y Alimentación) (2005), Evaluación Alianza para el Campo 2005, FAOSagarpa, México.

FAO-Sagarpa (Fondo de las Naciones Unidas para la Agricultura y la Alimentación-Secretaría de Agricultura, Ganadería, Pesca y Alimen- 
tación) (2007), Informe de evaluación de consistencia de resultados 2007, Alianza para el Campo, FAO-Sagarpa, México.

Friedmann, John (2001), Planificación en el ámbito público. Del conocimiento a la acción, Ministerio de Administraciones Públicas, Madrid.

GIADR (Grupo Interagencial de Desarrollo Rural-México) (2007), Temas prioritarios de politica agroalimentaria y desarrollo rural en México, GIADR, México.

Grindle, Marilee (2007), "Good enough governance revisited”, Development policy review, 25 (5), Londres, pp. 553-574.

Herrera Francisco, Bruno Lutz e Ivonne Vizcarra (2009), "La política de desarrollo rural en México y el cambio institucional 2000-2006", Economia, Sociedad y Territorio, vol. Ix, núm. 29, pp. 89-117.

Hewitt de Alcántara, Cynthia (1998), "Uses and abuses of the concept of governance", International Social Science Journal, 50 (155), Organización de las Naciones Unidas para la Educación, la Ciencia y la Cultura (Unesco), París, pp. 105-13.

Kaufmann Daniel, Aart Kraay and Massimo Mastruzzi (2010), The Worldwide gobernance indicators. Methodology and analytical issues, The World Bank, Washington.

Kooiman Jan y Martin van Vliet (2000), "Self-governance as a mode societal governance", Public Management: an international journal of research and theory, vol. 2 Issue 32, Tailor \& Francis LTD, Rotterdam, pp. 359-377.

LDRS (Ley de Desarrollo Rural Sustentable) (2001), "Ley de Desarrollo Rural Sustentable", Diario Oficial de la Federación, 7 de diciembre, México.

Mayntz, Renate (2000), "Nuevos desafíos de la teoría de governance", Instituciones y Desarrollo, 7, Institut Internacional de Gobernabilitat de Catalunya, Barcelona, pp. 35-51.

Moran Emilio, Elinor Ostrom and John Randolph (1998), A multinivel approach to studyng global environmental change in forest ecosystems, 
Center for the study of Institutions, populations an Enviromental Change, Indiana University Bloomington, Estados Unidos de América.

Paramio, Ludolfo (1994), "Gobernabilidad democrática, violencia y desigualdad en América Latina”, Cuadernos África América Latina 14 (94), SODEPAZ, Madrid, pp. 15-19

Pagden, Anthony (1998), "The genesis of governance and enlightenment conceptions of the cosmopolitan world order international", Social Science Journal 50 (155), Elsevier, Londres, pp. 7-15.

Parra Manuel y Obeimar Herrera (2005), Informe de seguimiento y evaluación del Proyecto de diversificación de la agricultura en México y Guatemala, Ecosur, San Cristóbal de las Casas, Chiapas.

Parra Manuel y Obeimar Herrera (2006), Fortalecimiento del Consejo Municipal de Desarrollo Rural Sustentable de Villaflores, Chiapas, México, Informe del proceso de colaboración Prosureste/GTzEcosur, San Cristóbal de las Casas, Chiapas.

Piñeiro, Martín (2009), La institucionalidad agropecuaria en América Latina: estado actual y nuevos desafios, FaO, Santiago de Chile.

Prats, Joan (2003), “El concepto y análisis de la gobernabilidad”, Revista instituciones y desarrollo No.14-15, Institut Internacional de Gobernabilitat de Catalunya, Barcelona, pp. 239-269.

Project Managment Institute (2004), Guía de los fundamentos de la dirección de proyectos, Project Managment Institute, Palo Alto.

Ruiz, Carlos (1996), Manual para la elaboración de políticas públicas, Plaza y Valdés Editores-Universidad Iberoamericana, México.

Sagarpa (Secretaría de Agricultura, Ganadería, Pesca y Alimentación) (2005), Modificaciones al marco normativo general contenidos en las reglas de operación de la Alianza para el Campo, Sagarpa, México.

Sierra, Restituto (1998), Técnicas de investigación social: teoría y práctica, Paraninfo, Madrid. 
Stalker, Linda (2005), "The relationship between participation and projects outcomes: evidence from rural water supply proyects in India", World Development, 33 (11), Elsevier, Londres, pp. 1801-1819.

Taylor Ellen, Boyd Rossing y Jean Gerand (1998), Evaluating collaboratives, reaching the potential, Wisconsin University, Cooperative extension, Madison.

Tellis, Winston (1997), "Introduction to case study", The Qualitative Report, 3 (2), Nova southeastern University, Florida, pp. 1-14.

Vázquez, José (2007), Informe de evaluación estatal. Programa de desarrollo rural Chiapas, Secretaría de Agricultura, Ganadería, Desarrollo Rural, Pesca y Alimentación-Gobierno del Estado de Chiapas, México.

шв (World Bank) (2000), Reforming public institutions and strengthening governance: A World Bank strategy, World Bank, Washington.

Yin, Robert (1981), "The case study crisis. Some answers", Administrative sciences Quarterly, 26 (1), Cornell University, Nueva York, pp. 58-65.

Recibido: 17 de diciembre de 2009. Reenviado: 8 de agosto de 2011. Aceptado: 19 de septiembre de 2011.

Obeimar Balente-Hererra. Es doctor ingeniero agrónomo en planificación y gestión de proyectos de desarrollo rural sostenible por la Universidad Politécnica de Madrid. Su adscripción laboral es el área de sistemas de producción alternativos de El Colegio de la Frontera Sur. Se ha especializado en los últimos años en desarrollo territorial e innovación. Entre sus últimas publicaciones destacan, en coautoria, "Estrategia regional de desarrollo comunitario del proyecto PADEs", en German Martínez (coord.), Tres estudios para el desarrollo sustentable en la selva Lacandona, Gobierno del Estado de Chiapas-Fondo de Población de las Naciones Unidas, Tuxtla Gutiérrez, Chiapas, pp. 205-301 (2008); en coautoría, "Modos de vida y seguridad alimentaría de los mayas de Campeche", Fondo de las Naciones Unidas para la Agricultura y la Alimentación, Roma, pp. 129-168 (2007); en coautoría (artículo ganador de la Iniciativa FAO América Latina y el Caribe sin hambre), Criterios de sustentabilidad para la 
planificación en los consejos municipales de desarrollo rural sustentable, $\mathrm{El}$ Colegio de la Frontera Sur-Agencia de Cooperación Alemana-Comisión Nacional de Áreas Naturales Protegidas, México (2007).

José María Díaz-Puente. Es doctor ingeniero agrónomo por la Universidad Politécnica de Madrid, con estancias de formación investigadora en Berkeley University, en el Institute of Urban and Regional Development (IURD), y Stanford University, en el Policy Analysis \& Evaluation Unit. Actualmente está adscrito a GESPLAN (Grupo I+D Planificación y Gestión del Desarrollo Rural-Local); departamento Proyectos y Planificación Rural; Universidad Politécnica de Madrid. Su línea de investigación actual es: evaluación del desarrollo. Sus publicaciones más recientes son: en coautoría, "Policy Support for the Diffusion of Innovation among sMEs: An Evaluation Study in the Spanish Region of Madrid", European Planning Studies, 17(3), Taylor \& Francis, Londres, pp. 365-387 (2009); en coautoría, "Empowering communities through evaluation: some lessons from rural Spain", Community Development Journal, 44 (1), Oxford Univesity Press, Oxford, pp. 53-67. (2009); en coautoría, "Building evaluation capacity in Spain: a case study of rural development and empowerment in the European Union", Evaluation Review, 32(5), sAGE publications, Thousand Oaks, pp. 478-506 (2008).

Manuel Roberto Parra-Vázquez. Es doctor en economía por la Universidad Nacional Autónoma de México. Investigador titular "C", adscrito al área de Sistemas de Producción Alternativos de El Colegio de la Frontera Sur. Sus líneas de investigación actuales son: desarrollo territorial y políticas públicas para el desarrollo local. Sus publicaciones más recientes son: en coautoría, "Planear participativamente: institucionalización de la participación social en el Consejo Distrital de Los Altos de Chiapas", en Trench Tim (coord.). La dimensión cultural en procesos de desarrollo rural regional: casos del campo mexicano, Universidad Autónoma Chapingo, México, pp. 65-110. (2008); en coautoría, "Estrategia regional de desarrollo comunitario del proyecto PADEs", en German Martínez (coord.) Tres estudios para el desarrollo sustentable en la Selva Lacandona, Gobierno del Estado de Chiapas-Fondo de Población de las Naciones Unidas, Tuxtla Gutierrez, pp. 205-301 (2008); en coautoría, Criterios de sustentabilidad para la planificación en los Consejos Municipales de Desarrollo Rural Sustentable, El Colegio de la Frontera Sur, México, (2007). 